\title{
Connection of thermopower, magnetothermopower with resistivity and magnetoresistance in manganites with $\mathrm{Nd}$ and Sm
}

Luidmila Koroleva ${ }^{1, *}$, Ivan Batashev ${ }^{1}$, Artem Morozov ${ }^{1}$, Anatolii Balbashov ${ }^{2}$, Henryk Szymczak ${ }^{3}$ and Anna SlavskaWanniewska ${ }^{3}$

${ }^{1}$ Moscow State University, 19991 Moscow, Russia

${ }^{2}$ Moscow Power Engineering Institute, 19991 Moscow, Russia

${ }^{3}$ Institute of Physics Polish Academy of Sciences, Warsaw, Poland

\begin{abstract}
Thermopower, magnetothermopower resistivity and magnetoresistance of single crystal samples $\operatorname{Re}_{(1-x)} \mathrm{Sr}_{x} \mathrm{MnO}_{3}(0 \leq x \leq 0.3, \mathrm{Re}=\mathrm{Nd}$, Sm) were studied in wide temperature interval, included the Curie temperature $T_{C}$. Giant maxima of resistivity and thermopower, very big negative magnetothermopower and magnetoresistance were found in $T_{C}$ region. So, a giant maximum of thermopower in $T_{C}$ was found, which suppressed by magnetic field. Simultaneous magnetic field destroys magneto-impurity states - ferrons as evidenced the maxima resistivity and magnetoresistance in $T_{C}$ Hence it follows that giant thermopower in manganites are produced by ferrons and its value is set by the quantity of impurities, that is the concentration of impurities and the volume of sample.
\end{abstract}

In our work, thermopower $S$, magnetothermopower $\Delta S / S$, electrical resistivity $\rho$, magnetoresistance $\Delta \rho / \rho$ and magnetization $M$ of the single crustal samples $\operatorname{Re}_{(1-}$ $\left.{ }_{x}\right) \mathrm{Sr}_{\mathrm{x}} \mathrm{MnO}_{3}(0 \leq \mathrm{x} \leq 0.3 ; \mathrm{Re}=\mathrm{Nd}, \mathrm{Sm})$ were studied. The temperature dependence of the magnetization $M(T)$ in different magnetic fields was measured by SQUIDmagnetometer. The obtained results show, that the transition from ferromagnetic to paramagnetic state is heavily blurred. From this, we conclude that the samples we studied are magnetically heterogeneous. They consist of ferromagnetic clusters located in A-type antiferromagnetic matrix. Curie temperature $T_{C}$ was defined as the temperature of minimum on $d M(T) / d T$ curve, measured in magnetic field $0.1 \mathrm{kOe}$. In this work the giant negative magnetoresistance was observed in these compounds near $T_{C}$. This indicates that these ferromagnetic clusters are of ferron type [1]. A sharp increase of $S$ and module of negative $\Delta S / S$ were discovered near $T_{C}$, where $\Delta S / S_{1}$ reaches giant values up to $94.5 \%$ in magnetic field $13.2 \mathrm{kOe}$ in case of compound with $\mathrm{Re}=\mathrm{Sm}, \mathrm{x}=0.3$. In Fig. 1 the temperature dependence of thermopower $S(T)$ in magnetic field is shown for $\mathrm{Nd}$ compound with $x=0.3$.

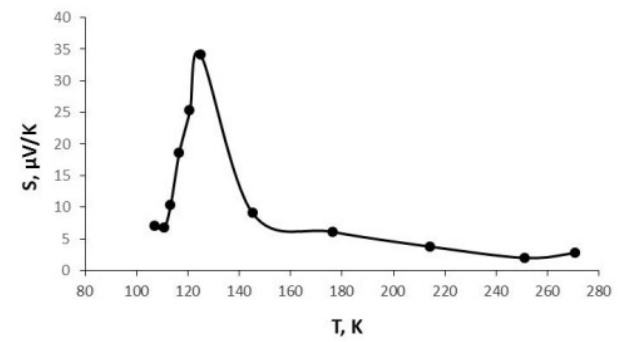

Fig. 1 Temperature dependence of $\mathrm{S}(T)$ for $\mathrm{Nd}_{0.7} \mathrm{Sr}_{0.3} \mathrm{MnO}_{3}$.
Figure 2 shows the temperature dependence of magnetothermopower $\{\Delta S / S\}(T)$ for this sample in different magnetic fields.

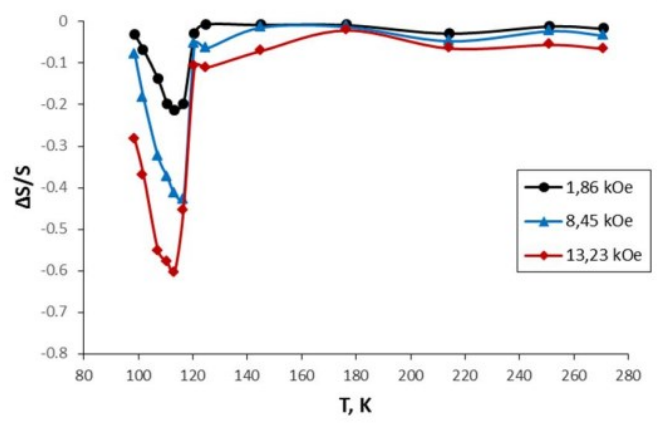

Fig. 2. Temperature dependence of magnetothermopower $\{\Delta S / S\}(T)$ in different magnetic fields for $\mathrm{Nd}_{0.7} \mathrm{Sr}_{0.3} \mathrm{MnO}_{3}$

It's clear that magnetothermopower is negative and its absolute value near $T_{C}$ reaches a giant value of $60 \%$ in a magnetic field $13.23 \mathrm{kOe}$. A similar behavior of the thermopower and magnetothermopower was observed in all compounds we researched. The curves presented above are typical for all examined compounds. Maximum values for all samples are shown in table 1 . Also in table 1 maximum values of specified resistivity $\rho$ and $\Delta \rho / \rho$ are shown. It should be noted that in compounds with $x=0$ the value of $S$ is very small. This means, that thermopower is caused by presence of ferrons, as the value of the thermopower falls with the destruction of ferrons caused by magnetic field or heating to temperatures over $T_{C}$. Inside ferrons the crystalline lattice is compressed as discussed in $[2,3]$, so it is possible to assume that the value of thermopower inside is different from thermopower in antiferromagnetic matrix. In this case thermopower $S_{I}$ appears on every single ferron under the influence of

\footnotetext{
Corresponding author: koroleva@phys.msu.ru
} 
local temperature gradient $\Delta T_{l}$, caused by total temperature gradient $\Delta T$, as shown in Fig. 3. Each such microregion contributes $\left(S_{1}-S_{2}\right)$ to the thermopower of the whole sample. Where $S_{2}$ is the thermopower of AFM matrix without ferrons.

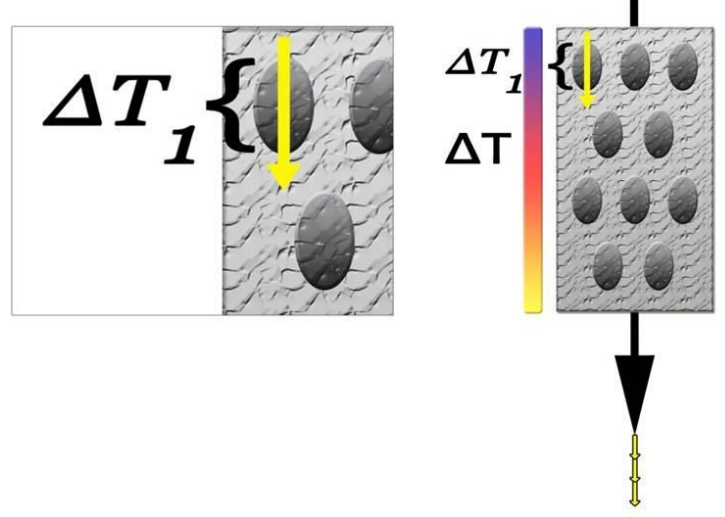

Fig. 3 Schematic representation of ferrons (ellipsoids) and their role in creation of thermopower in magnetic semiconductors.
So, in doped magnetic semiconductors thermopower is determined by number of ferrons and, therefore, by level of doping and volume of the sample. This was confirmed by changing the volume of sample. After dividing the sample in two parts thermopower of each part decreased proportionally the volume of the part. Thus, in doped magnetic semiconductors the value of thermopower can be considerably increased by enlarging the volume of sample, the level of doping and can be controlled by magnetic field.

Thus, in doped magnetic semiconductors, which include manganites, the value of thermopower can be significantly increased in comparison with undoped one, by increasing the concentration of impurities and the volume of the sample. Furthermore, thermomaterials based on magnetic semiconductors should be characterized by specific thermopower, that is the value of thermopower per unit volume. Exactly these values are shown in table 1 , which are significantly different from those shown in the Fig. 1.

Table 1. Thermopower $S$, magnetothermopower $\Delta \mathrm{S} / \mathrm{S}$, electroresistivity $\rho$, magnetoresistance $\Delta \rho / \rho$ and Curie temperature for manganites $\mathrm{Nd}_{(1-\mathrm{x})} \mathrm{Sr}_{\mathrm{x}} \mathrm{MnO}_{3}$ and $\mathrm{Sm}_{(1-\mathrm{x})} \mathrm{Sr}_{\mathrm{x}} \mathrm{MnO}_{3}$.

\begin{tabular}{|l|l|l|l|l|l|}
\hline Compound & $T_{C}, \mathrm{~K}$ & $S, \mu \mathrm{V} /\left(\mathrm{K} \cdot \mathrm{cm}^{3}\right)$ & $\Delta S / S, \%$ & $\rho, \mathrm{k} \Omega \cdot \mathrm{cm}$ & $\Delta \rho / \rho, \%$ \\
\hline $\mathrm{Sm}_{0.85} \mathrm{Sr}_{0.15} \mathrm{MnO}_{3}$ & 80 & 525 & 54 & 168 & 70 \\
\hline $\mathrm{Sm}_{0.8} \mathrm{Sr}_{0.2} \mathrm{MnO}_{3}$ & 90 & 575 & 37 & 208 & 33 \\
\hline $\mathrm{Sm}_{0.75} \mathrm{Sr}_{0.25} \mathrm{MnO}_{3}$ & 95 & 900 & 64 & 260 & 51 \\
\hline $\mathrm{Sm}_{0.7} \mathrm{Sr}_{0.3} \mathrm{MnO}_{3}$ & 87 & 210 & 94 & 67.7 & 76 \\
\hline $\mathrm{Nd}_{0.7} \mathrm{Sr}_{0.3} \mathrm{MnO}_{3}$ & 110 & 86 & 15 & 25 & 34 \\
\hline $\mathrm{Nd}_{0.8} \mathrm{Sr}_{0.2} \mathrm{MnO}_{3}$ & 155 & 240 & 42 & 70 & 45 \\
\hline $\mathrm{Nd}_{0.85} \mathrm{Sr}_{0.15} \mathrm{MnO}_{3}$ & 120 & 1042 & 34 & 60 & 25 \\
\hline $\mathrm{NdMnO}_{3}$ & - & $\leq 0,2 \mu \mathrm{V} / \mathrm{K}$ & - & - & - \\
\hline
\end{tabular}

\section{References.}

1. E. L. Nagaev. Phys. Rep. 346, 387-531, (2001)

2. T. Kasuya, A. Yanase. Rev. Mod. Phys. 40, 684696, (1968).

3. T. Kasuya, A. Yanase. J. Phys. Soc. Jap. 25, $1025-$ 1042, (1968) 